G. Coates, et al., Int. J. of Safety and Security Eng., Vol. 6, No. 3 (2016) 627-635

\title{
INVESTIGATING THE FLOOD RESPONSIVENESS OF SMALL AND MEDIUM ENTERPRISES USING AGENT- BASED MODELLING AND SIMULATION
}

\author{
G. COATES ${ }^{1}$, C. LI $^{1}$, N.G. WRIGHT ${ }^{2} \&$ S. AHILAN ${ }^{3}$ \\ ${ }^{1}$ Durham University, United Kingdom. \\ ${ }^{2}$ De Montfort University, United Kingdom. \\ ${ }^{3}$ University of Leeds, United Kingdom.
}

\begin{abstract}
In recent years, flooding has caused major damage and disruption to businesses in the United Kingdom (UK) resulting in significant losses to the economy. The effect of flooding on Small and Medium Enterprises (SMEs) is of particular importance given this size of business represents a major component of the UK's economy. More specifically, according to the UK's Department for Business Innovation and Skills, SMEs account for $99.9 \%$ of all private sector businesses and approximately $47 \%$ of annual turnover. In contrast to large businesses, SMEs often have limited resources and in most cases lack business continuity plans, which diminishes the effectiveness and efficiency of their response to flooding. This paper presents an overview of an approach taken to agent-based modelling and simulation of manufacturing SMEs experiencing a flood event, and related organisations. In this overview, the sources used to develop the attributes and behaviour of SMEs are highlighted. In addition, the paper presents preliminary results of simulations of the behaviours and actions of manufacturing SMEs in response to the 2007 flood event in the Lower Don Valley region of Sheffield in the UK. Results include key performance metrics of SMEs which provide an indication of the effectiveness and efficiency of the response of the businesses modelled.
\end{abstract}

Keywords: agent-based modelling and simulation, flooding, small businesses.

\section{INTRODUCTION}

Flooding is the most common and widespread natural disaster in the UK [1]. In addition to households being affected by flooding, businesses can also suffer and experience significant losses in terms of damage to property and disruption to operations. For example, in terms of losses to UK businesses, the Environment Agency (EA) estimated the financial cost of the 2007 and 2012 floods at approximately $£ 740$ million and $£ 600$ million respectively [1]. The impact of flooding is arguably more acutely felt by small and medium enterprises (SMEs) due to their lack of resources, planning and organisational resilience in the face of such disruptive events [2-3]. The European Union classifies SMEs according to the number of employees and turnover or balance sheet total [4]. In terms of number of employees, micro-businesses have less than 10, small-sized have greater than or equal to 10 but less than 50, and medium-sized have greater than or equal to 50 but less than 250. In the UK, $99.9 \%$ of private sector businesses are SMEs accounting for approximately $60 \%$ of employment and $47 \%$ of annual turnover [5]. Table 1 presents a breakdown of these figures according to SME size. Furthermore, Table 1 shows that micro-businesses dominate in terms of proportion of SME businesses, employment and turnover. 
Table 1: Breakdown of private sector SMEs in the UK [5].

\begin{tabular}{lccc}
\hline SMEs & Businesses (\%) & Employment (\%) & Turnover (\%) \\
\hline Micro (1-9 employees) & 95.5 & 32.7 & 18.1 \\
Small (10-49 employees) & 3.8 & 15.3 & 14.6 \\
Medium (50-249 employees) & 0.6 & 12.3 & 14.5 \\
Total & $\mathbf{9 9 . 9}$ & $\mathbf{6 0 . 3}$ & $\mathbf{4 7 . 2}$ \\
\hline
\end{tabular}

With its emphasis on SMEs, given the composition of UK business and significance of companies of this size, the UK's Engineering and Physical Sciences Research Council funded SESAME project aims to explore and improve the ways in which these businesses prepare for and respond to flooding thus making them more resilient to these disruptive events [6]. While the SESAME project consists of a multi-disciplinary team of researchers pursuing a number of research goals, this paper focuses on the computational modelling and simulation framework developed to facilitate an investigation into how the behaviours, activities and actions of these SMEs influence the continuity of their business operations during and in the aftermath of flood events.

The remainder of this paper is organised as follows. Section 2 presents a brief synopsis of related work. In Section 3, an overview of the modelling and simulation framework is given. Section 4 presents a preliminary case study related to the flooding of Sheffield's Lower Don Valley. Furthermore, for illustrative purposes, a simulation involving an analysis of SMEs with and without potential behaviours is considered. Finally, conclusions and future work are provided in Section 5.

\section{RELATED WORK}

Research into modelling the preparedness, response and recovery of SMEs to flooding is scarce. SMEs' responses to flood events, along with recovery post flooding, are complex processes requiring a suitable modelling approach. One approach to modelling such processes is agent-based modelling. This approach has received increasing attention in recent years to facilitate the study of complex systems, and it has been applied to a range of fields such as business and social science [7]. Hitherto, several agent-based models have been developed to simulate either flood risk related to individuals $[8,9]$ or business aspects of SMEs $[10,11]$. As examples, an agent-based model has been developed to understand the process of growth and innovation that evolves in SMEs over time [10], while another ABM has been developed to study supply chains involving SMEs [11]. Despite this research on agent-based models in the context of small businesses, modelling SMEs in the context of disruptive events, in particular flood events, remains largely unexplored.

\section{MODELLING AND SIMULATION FRAMEWORK}

In order to investigate SMEs' behaviour when faced with flooding, agent-based modelling and simulation (ABMS) using the Repast platform [12], allied with flood modelling, has been employed. Achieving this aim has involved developing: (i) the agent-based model's (ABM's) virtual geographic environment (VGE), which is able to combine Ordnance Survey information with flood model output, to identify the businesses affected by flooding [13]; (ii) agents to model businesses in terms of their attributes, behaviour, actions and interactions in response to flood events [14]; (iii) an agent interaction network to enable simulations to be performed. An overview of the modelling and simulation framework is presented in Fig. 1 and described in further detail in [13]. 


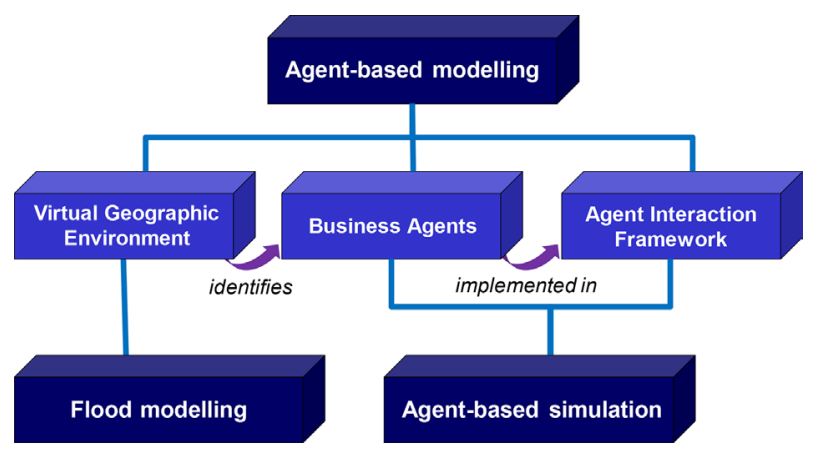

Figure 1: Overview of modelling and simulation framework [13].

\section{PRELIMINARY CASE STUDY}

In the context of the SESAME project, the Lower Don Valley region of Sheffield has been identified as a suitable case study area due to its high concentration of SMEs from a range of sectors, particularly manufacturing, allied with their experience of significant flooding in 2007. In June 2007, approximately $100 \mathrm{~mm}$ of rainfall fell in 24 hours in the Lower Don Valley region having a devastating impact on more than 1,000 businesses including key manufacturing companies with one suffering over $£ 15$ million worth of damage [15].

\subsection{Flood modelling}

A one in 1,000 year flood event has been simulated for the River Don in the Lower Don Valley region of Sheffield, which is similar in scale to the flood event experienced in June 2007. The simulation used a hydrodynamic model to obtain flood depths at half hour intervals over the flood duration. The 1D-2D ISIS-TUFLOW hydrodynamic model of the River Don provided by the EA has been used. The 1D ISIS model represents the Don River channel whereas the 2D TUFLOW model represents the surrounding floodplain and urban areas. In recent years, rainfall run-off boundaries have been improved with the introduction of the Revitalised Flood Hydrograph (ReFH) methodology. One in 1,000 year flood hydrographs were (i) used as inflow units to the hydrodynamic model and (ii) developed in compliance with recent guidelines produced by the EA in which the ratios of the ReFH are multiplied by the statistical 100 year estimates to gain the 1,000 year estimate.

Simulation results for a one in 1,000 year flood event are shown in Fig. 2 at 10 hour time intervals over a 40 hour period. For the case study of the River Don Valley in Sheffield, the dynamic flood inundation data associated with the maps shown in Fig. 2, and at other time intervals, provide the input for the ABM's VGE, which, in turn, provides input for agentbased simulations. That is, in agent-based simulations, the actions and interactions of agents representing flooded SMEs are driven by the depth of water at their respective premises, and adjacent roads, at different times throughout the flood event being modelled. An example of VGE output is shown in Fig. 3a, which is based on the inundation data for Sheffield at $t=20$ hours corresponding to Fig. 2b. Although only four maps are shown in Fig. 2, hydrodynamic modelling has provided estimated water depths at regular time intervals, i.e. each half hour, during the simulated flood event.

The VGE is constructed using Geographic Information System (GIS) data from OS MasterMap's Topography Layer, Integrated Transport Network ${ }^{\mathrm{TM}}$ Layer and Address Layer 2 


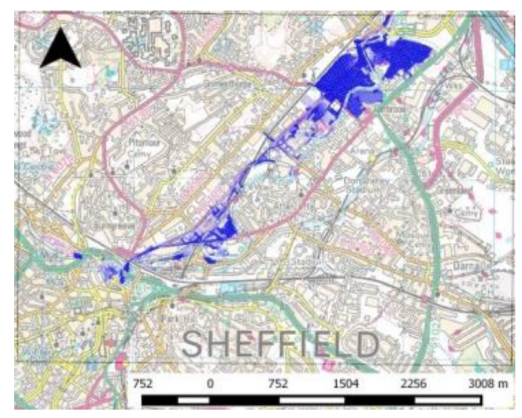

(a) 1000 year flood extent at $t=10$ hours

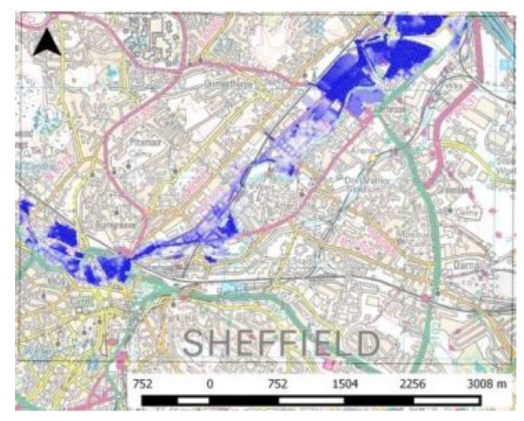

(c) 1000 year flood extent at $\mathrm{t}=30$ hours

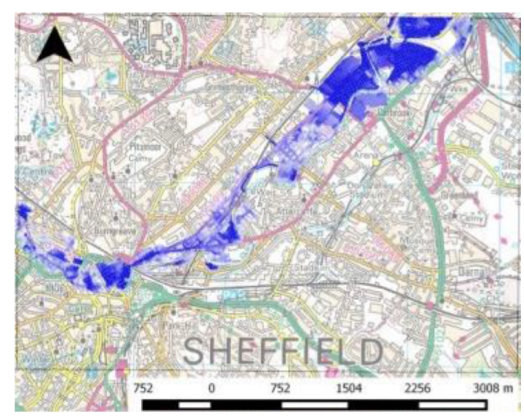

(b) 1000 y ear flood extent at $t=20$ hours

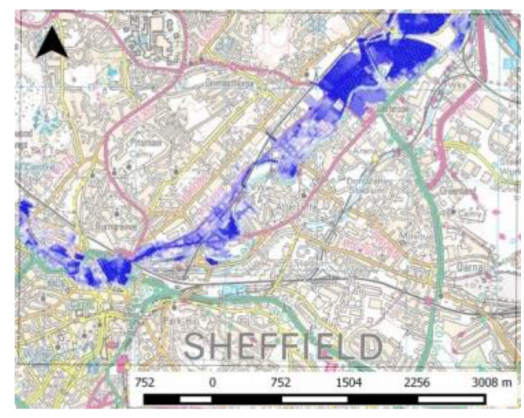

(d) 1000 year flood extent at $\mathrm{t}=40$ hours

Figure 2: Flood inundation maps for Sheffield's lower don valley.

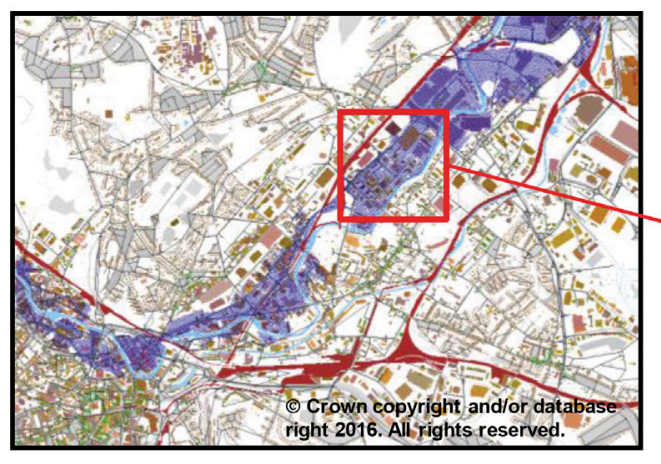

(a)

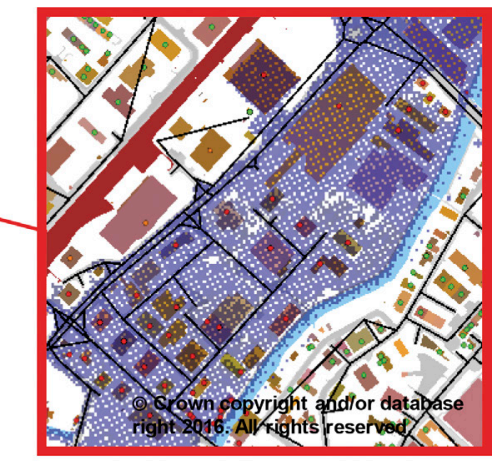

(b)

Figure 3: Sample VGE output for the Lower Don Valley in Sheffield.

[16]. The Topography Layer provides information on individual buildings; the Integrated Transport Network ${ }^{\mathrm{TM}}$ (ITN) Layer provides information on the road network; the Address Layer 2 provides information on commercial properties including the precise location and the identification of the associated building in the Topography Layer and road link in the ITN Layer. In addition to showing the extent of flood water displayed in the VGE for the case study area under consideration, Fig. $3 b$ indicates the location of each business using a 
circle symbol, which is colour-coded according to whether their associated building is flooded (red), or their building is not flooded but their associated road link is flooded (orange), or neither their associated building nor road link is flooded (green). Throughout a simulated flood event, the status of each business modelled varies according to the dynamic inundation data.

The ABM's VGE software classifies businesses and organisations according to the National Land Use Database (NLUD) code, which consists of 41 groups, within the Address Layer 2 of OS MasterMap. An analysis of Sheffield's Lower Don Valley region has been performed using the VGE software to identify the types of businesses in this area. The VGE identified 5,570 business/organisations with retail (U091 for shops, U092 for financial and professional services) along with industry and business (U101 for manufacturing, U102 for offices) dominating. This analysis provides input for the ABM in terms of identifying the precise geographic location of each SME along with their type and, based on inundation data for the particular flood scenario considered, which of these businesses experiences flooding and to what extent in terms of depth of water and length of time before the water recedes.

\subsection{Agent-based modelling}

In the context of our work, agent-based modelling has involved capturing and representing the attributes, actions, interactions and dynamic behaviours of SMEs, and related organisations, such that simulation can be used to assess ways in which SMEs can improve their response to flood events. With a focus on SMEs from the manufacturing sector, agents have been developed to model small businesses in terms of their behaviour and attributes relevant to responding to and recovering from flood events. Simultaneously, an agent interaction network has been developed containing agents representing other related organisations such as suppliers, customers and service companies.

SME agents' attributes and behaviours have been derived from a number of sources: (a) information extracted from transcripts of semi-structured interviews with SMEs at risk of flooding and/or which have experienced flooding; (b) discussions with colleagues who conducted the aforementioned interviews to ensure the attributes and behaviours, and the duration range of behaviours along with the relationships between them, being used to model SMEs are appropriate and relevant; (c) EA literature providing advice for businesses preparing for flooding; (d) literature detailing business continuity management (BCM) systems' requirements (ISO223301). In this work, SME behaviours extracted from interview transcripts are referred to as 'existing behaviours' whereas those found in EA and BCM literature are referred to as 'potential behaviours'. This distinction is made as it provides the basis for a comparative analysis in our modelling environment in terms of what SMEs did when flooded in the past and what they may do in the future.

In addition to modelling the attributes and behaviours of SMEs, simulations of the behaviours of business agents during and in the short-term aftermath of a flood event has required the development of other agents to model organisations such as suppliers, customers, mutual aid partners and service companies. Furthermore, relationships between SMEs and these organisations have been modelled since some will influence the response and recovery of an SME when a flood event occurs. For example, the existence of a pre-agreed priority relationship between an SME and a service company may lead to preferential treatment thus expediting recovery and resumption of normal operations. Similarly, the existence of a pre-existing mutual aid agreement between SMEs may enable the flood-affected SME to 
resume some degree of production operations, in the case of manufacturing SMEs, thus enabling customer demands to be met to a greater degree than if such an arrangement was not in place.

\subsection{Agent-based simulation}

Simulating SMEs during and in the short-term aftermath of flood events provides a means of enabling what-if scenarios/experiments to be performed to investigate the effect of different behaviours on operational response and business continuity. In terms of agent-based simulations, we are currently in the process of designing experiments for simulations which will enable us to assess the effect of different combinations of behaviours on the performance of the small businesses modelled. However, as part of the process of developing SME agents incorporating existing and potential behaviours, preliminary simulations have been conducted to assess the functionality of the associated software. This paper presents some results from those preliminary simulations.

For illustrative purposes, a preliminary simulation involving manufacturing SMEs is considered. Furthermore, the SMEs considered are micro-sized since, as indicated in Section 1, this size of business accounts for the vast majority, i.e. $95.5 \%$ of $99.9 \%$, of SMEs in the UK. In response to the flood event (tick 56-137) in Sheffield's Lower Don Valley as described in Section 4.1, Fig. 4 shows the average production capacity level of all manufacturing microsized SMEs modelled for 20 repeat simulations: (a) without potential behaviours; (b) with some potential behaviours. Also, in Fig. 4, the simulation time of 1,200 ticks corresponds with 25 days since there are 48 ticks per day, i.e. one tick per half hour.

In terms of what is meant by 'with some potential behaviours', in addition to the SMEs being registered to receive alerts from the EA, they have also raised the level/height at which their machines, raw materials, paper documents and electrical sockets are located to mitigate damage should a flood event occur. Each of these potential behaviours has associated benefits such as being registered to receive alerts from the EA provides an SME with time to take preparatory action in terms of, for example, lifting up IT equipment and taking measures to stop the flood water entering premises before employees are evacuated. Raising the levels of machines, raw materials and electrical sockets lessens the damage to them, whereas raising

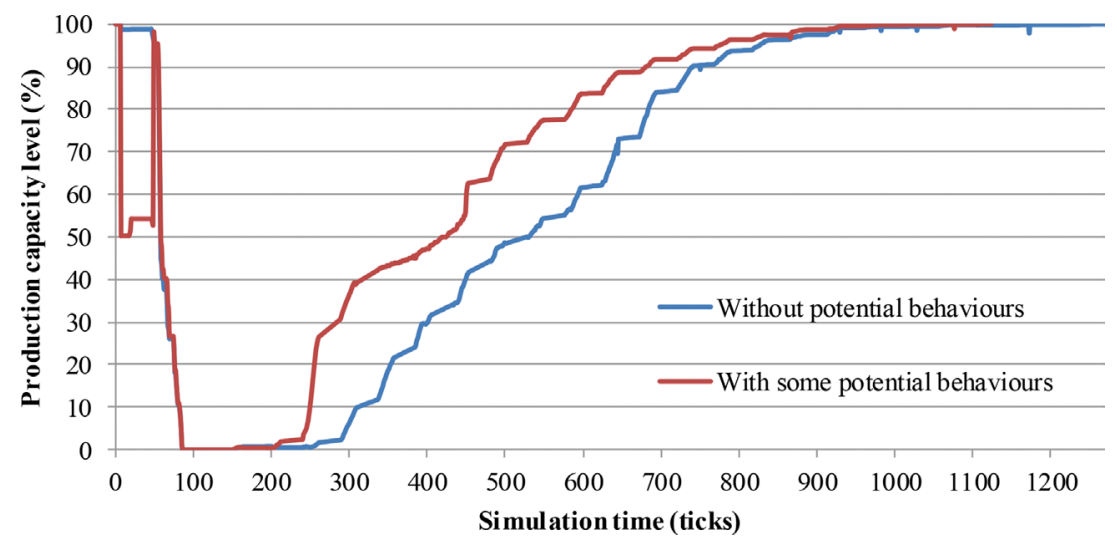

Figure 4: Simulation output for all manufacturing micro-sized SMEs. 
paper documents enables an SME to contact service companies, customers and suppliers more quickly than if these documents were damaged. In Fig. 4, it can be seen that manufacturing SMEs with the potential behaviours mentioned, are able to achieve a greater production capacity level earlier than those SMEs which do not have these behaviours. Also, in relation to Fig. 4, the production capacity level, $P C_{\text {level }}$, at each time interval, i.e. each tick signifying half an hour, is determined using the following equation,

$$
P C_{\text {level }}=R_{\text {level }}^{e} \times R_{\text {level }}^{m}
$$

given there is a power supply to the premises and there is sufficient raw materials available for this level of production, and where $R_{\text {level }}^{e}$ is ratio of an SME's available employees to total employees, and $R_{\text {level }}^{m}$ is the ratio of an SME's available machines to total machines. To note, at each time interval, one unit of raw materials is consumed given (i) at least this amount is available, (ii) all employees are available and utilised, and (iii) all machines are available and utilised.

In the same simulation associated with the data shown in Fig. 4, the average production capacity level of a single manufacturing micro-sized SME for 20 repeat simulations is shown in Fig. 5.

In a typical simulation for this SME without the aforementioned potential behaviours, once the flood water approached the SME's premises, employees evacuated before water entered the property reaching a height of 0,913 metres. During the flood, power was lost and a proportion of the SME's machines were damaged and raw materials ruined due to the water depth reached. Employees returned to the premises only when the flood water had receded and thus recovery operations could begin. However, due to the flood event, with some machines inoperable and raw materials damaged, the SMEs' ability to manufacture products, once the flood water had receded, was degraded. Furthermore, during the immediate recovery period, employees returning to the premises carried out activities such as cleaning and salvaging raw materials, contacting the insurance company and electrical service company. After an electrician attended to restore power to the premises and the insurance company organised a cleaning contract company to clean-up the premises, the SME carried out other activities including cataloguing damage, repairing machinery, cleaning-up the storage area for raw materials, ordering raw materials, and contacting suppliers and customers. Manufacturing products only resumed at the beginning of the third day after the flood ( tick 241) once employees were available for production,

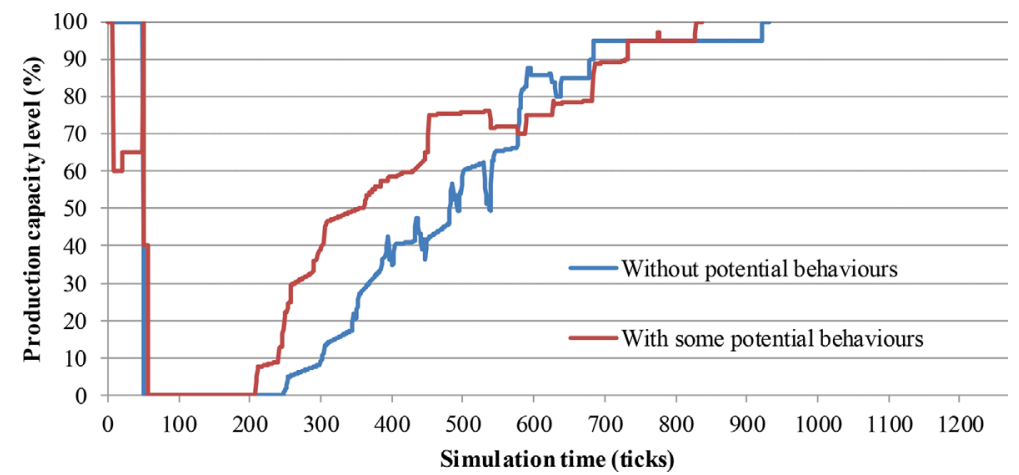

Figure 5: Simulation output for a single manufacturing micro-sized SME. 
machines were repaired and raw materials available. In contrast, while similar activities were undertaken in a typical simulation for this SME with the aforementioned potential behaviours, the company was able to resume production during the second day after the flood ( tick 207).

\section{CONCLUSIONS AND FUTURE WORK}

Flooding continues to cause businesses to suffer significant losses in terms of damage to property and disruption to operations. Given the significance of SMEs to the economy, research aimed at understanding how these businesses behave and how they may make changes to their behavior to better respond and recover from flood events may provide an insight to improving their business continuity.

In the strand of the SESAME project reported in this paper, research has focused on modelling existing, or typical, behaviours of SMEs in response to flooding and potential, or enhanced, behaviours aimed at improving their response. Based on data obtained from the field, an agent-based model has been developed to enable the impact of potential behaviours to be assessed. A preliminary case study focussing on manufacturing SMEs experiencing a one in 1,000 year flood event in the Lower Don Valley region of Sheffield has indicated the benefit of registering for EA alerts and raising machines, raw materials, paper documents and electrical sockets in terms of expediting a return to production operations. Future work will focus on the design of experiments to simulate SMEs during and in the aftermath of flood events to allow the investigation of the impacts of the range of potential behaviours on operational response and business continuity. Consequently, an assessment will be made as to which combination of behaviours may be most suitable for SMEs to implement in order to improve business continuity.

\section{ACKNOWLEDGEMENTS}

The authors gratefully acknowledge the funding provided be the UK's EPSRC under grant $\mathrm{EP} / \mathrm{K} 012770 / 1$. The data used in the preliminary case study presented in this paper is unavailable due to it including data from OS MasterMap's Address Layer 2 obtained under a research agreement.

\section{REFERENCES}

[1] http://www.environment-agency.gov.uk

[2] McGuinness, M. \& Johnson, N., Responding to changing paradigms of risk: managing flood risk and enhancing organisational resilience. In Proceeding of the Association of Geographical Societies in Europe (EUGEO) 2013 Congress, 'Changing Geographies and Geographies of Change', Rome, Italy, 2013.

[3] Reuter, C., Towards efficient security: business continuity management in small and medium enterprises. International Journal of Information Systems for Crisis Response and Management, 7(3), pp. 69-79, 2015.

[4] http://ec.europa.eu/growth/smes/business-friendly-environment/sme-definition/index _ en.htm

[5] Rhodes, C., Business statistics, briefing paper 06152, house of commons library, 2015.

[6] Coates, G., Hawe, G.I., McGuinness, M., Wright, N.G., Guan, D., Harries, T. \& McEwen, L., A framework for organisational operational response and strategic decision making for long term flood preparedness in urban areas'. In Proceeding of the 3rd International Conference on Disaster Management, A Coruña, Spain, 2013. 
[7] Heath, B., Hill, R. \& Ciarallo, F., A survey of agent-based modeling practices (January 1998 to July 2008). Journal of Artificial Societies and Social Simulation, 12(4), p. 9 , 2009.

[8] Dawson, R.J., Peppe, R. \& Wang, M., An agent-based model for risk-based flood incident management. Natural Hazards, 59, pp. 167-189, 2011.

[9] Liu, Y., Okada, N., Shen, D. \& Li, S., Agent-based flood evacuation simulation of lifethreatening conditions using vitae system model. Journal of Natural Disaster Science, 31, pp. 69-77, 2009.

[10] Blackmore, K., Bossomaier, T., Jarratt, D. \& Nesbitt, K., Intelligent agent framework for modelling the evolution of small and medium sized enterprises. In Proceeding of the 8th Australian and New Zealand Conference on Intelligent Information Systems, Sydney, Australia, 2003.

[11] Tounsi, J., Habchi, G., Boissière, J. \& Azaiez, S., A multi-agent knowledge model for SMEs mechatronic supply chains. Journal of Intelligent Manufacturing, 23, pp. 26472665, 2012.

[12] http://repast.sourceforge.net/

[13] Coates, G., Hawe, G.I., Wright, N.G. \& Ahilan, S., Agent-based modelling and inundation prediction to enable the identification of businesses affected by flooding'. In Proceeding of the 4th International Conference on Flood Recovery, Innovation and Response, Poznan, Poland, 2014.

[14] Li, C. \& Coates, G., Design and development of an agent-based model for business operations faced with flood disruption. International Journal of Design \& Nature and Ecodynamics, 11(2), pp. 97-106, 2016.

[15] Environment Agency, Review of 2007 summer floods, December 2007.

[16] http://www.ordnancesurvey.co.uk 